\title{
Perceived social support and the risk of cardiovascular disease and all- cause mortality in the Women's Health Initiative Observational Study
}

\author{
Nancy Freeborne, PA-C, MPH, DrPH, ${ }^{1}$ Samuel J. Simmens, PhD, ${ }^{2}$ JoAnn E. Manson, MD, DrPH, ${ }^{3}$ \\ Barbara V. Howard, PhD, ${ }^{4}$ Crystal W. Cené, MD, MPH, ${ }^{5}$ Matthew A. Allison, MD, MPH, ${ }^{6}$ \\ Giselle Corbie-Smith, MD, MSc, ${ }^{7}$ Christina L. Bell, MD, ${ }^{8}$ Natalie L. Denburg, PhD, ${ }^{9}$ \\ and Lisa Warsinger Martin, MD ${ }^{10}$
}

\begin{abstract}
Objective: Previous studies have shown social support to be inversely associated with cardiovascular disease (CVD) in men, whereas fewer studies have assessed the relationship in women. The purpose of this study was to evaluate the relationship between perceived social support and cardiovascular outcomes among postmenopausal women enrolled in the Women's Health Initiative Observational Study.

Methods: We examined the relationships between perceived social support and (1) incident coronary heart disease (CHD), (2) total CVD, and (3) all-cause mortality. Participants were Women's Health Initiative Observational Study women, ages 50 to 79 years, enrolled between 1993 and 1998 and followed for up to 10.8 years. Social support was ascertained at baseline via nine questions measuring the following functional support components: emotional/informational, tangible, positive social interaction, and affectionate support.

Results: Among women with prior CVD $(n=17,351)$ and no prior $\operatorname{CVD}(n=73,421)$, unadjusted hazard ratios ranged from 0.83 to 0.93 per standard deviation increment of social support. Adjustment for potential confounders, such as smoking and physical activity levels, eliminated the statistical significance of the associations with CHD and CVD. However, for all-cause mortality and among women free of baseline CVD, the association was modest but remained statistically significant after this adjustment (hazard ratio $=0.95[95 \%$ confidence interval, 0.91-0.98]). No statistically significant association was observed among women with a history of CVD.

Conclusions: After controlling for potential confounding variables, higher perceived social support is not associated with incident CHD or CVD. However, among women free of CVD at baseline, perceived social support is associated with a slightly lower risk of all-cause mortality.

Key Words: All-cause mortality - Cardiovascular disease - Postmenopausal - Social support.
\end{abstract}

$\mathrm{R}$ ates of cardiovascular disease (CVD) morbidity and mortality have declined in the past few decades, ${ }^{1}$ yet CVD still remains a primary cause of death in Americans, resulting in approximately one third of all deaths. ${ }^{1}$ Traditionally, more men than women were diagnosed with CVD, but in recent years, women's rates of CVD have

Received August 2, 2018; revised and accepted November 12, 2018. From the ${ }^{1}$ Department of Health Administration and Policy, George Mason University, Fairfax, VA; ${ }^{2}$ Department of Epidemiology and Biostatistics, Milken Institute School of Public Health, George Washington University, Washington, DC; ${ }^{3}$ Division of Preventive Medicine Brigham and Women's Hospital, Professor of Medicine, Harvard Medical School, Boston, MA; ${ }^{4}$ Senior Scientist, Medstar Health Research Institute, Washington, DC; ${ }^{5}$ Department of Medicine, Division of General Internal Medicine, University of North Carolina, Chapel Hill, NC;

Department of Family Medicine and Public Health, University of California San Diego, San Diego, CA; ${ }^{7}$ Department of Social Medicine, Department of Medicine, University of North Carolina School of Medicine, Chapel Hill, NC; ${ }^{8}$ University of Hawaii, Honolulu, HI; ${ }^{9}$ Department of Neurology, University of Iowa, Iowa City, IA; and ${ }^{10}$ Division of Cardiology, School of Medicine and Health Sciences, George Washington University, Washington, DC. increased to close to men's rates (35.9\% of American women have CVD vs $37.4 \%$ of men). ${ }^{1}$ Researchers have predominantly assessed the influence of traditional risk factors such as smoking and hypertension on CVD, but evidence that psychosocial factors can affect CVD has been promising and should be further evaluated. ${ }^{2,3}$ Because menopausal women

Funding/support: The Women's Health Initiative program was funded by the National Heart, Lung, and Blood Institute of the National Institutes of Health, Department of Health and Human Services through contracts HHSN268201100046C, HHSN268201100001C, HHSN268201100002C, HHSN268201100003C, HHSN268201100004C, and HHSN271201100004C.

Financial disclosures/conflicts of interest: None reported.

Supplemental digital content is available for this article. Direct URL citations appear in the printed text and are provided in the HTML and PDF versions of this article on the journal's Website (www.menopause.org).

Address correspondence to: Nancy Freeborne, PA-C, MPH, DrPH, George Mason University, Department of Health Administration and Policy, 4400 University Drive, MS 1J3 Peterson Hall , 4400D Fairfax, Virginia 22030. E-mail: NFreebor@gmu.edu 


\section{Support Type}

\author{
Functional/Resources
}

Network Structure
Typical Measurement

Perceived support

Received support

Network size

Social ties

\author{
$\underline{\text { Related Concepts }}$ \\ -Family relationships, presence of children \\ -Marital status \\ -Emotional well-being, depression \\ -Religiosity
}

FIG. 1. Overview of social support and related concepts. Cohen S, Underwood LG, Gottlieb BH eds. Social Support Measurement and Intervention: A Guide for Social Scientists. New York: Oxford University Press; 2000. Related concepts may function as antecedents or consequences of social support or both, or may be correlated with social support types for other reasons.

have moderate risks for developing CVD, establishing additional prevention efforts will be helpful.

Over the last three decades, greater scientific attention has been given to the relationship between psychosocial factors and health, particularly cardiovascular health, yet the relationship between social support and CVD needs further elucidation. Risk factors that have a social element, such as loneliness, or a psychological element, such as depression, are associated with disease such as $\mathrm{CVD}^{4,5}$; breast cancer recurrence $^{6}$; symptoms $^{7,8}$; health behaviors ${ }^{9,10}$; and mortality. ${ }^{11-13}$ Psychosocial factors association with CVD have been found $^{14-19}$ and psychosocial interventions have been suggested as adjuvant to more traditional treatments such as medical management and health behavior change efforts. ${ }^{2,3}$

There are multiple views and measures of social support. ${ }^{20}$ Typically, social support has been conceived as (1) the network of people connected to an individual (structural support) or (2) the resources available to an individual, such as help with a chore (functional support). ${ }^{20,21}$ Social networks or ties have been associated with health ${ }^{22}$ and mortality ${ }^{23}$ and pertaining to this study, related to CVD risk factors, ${ }^{24-26}$ incident or prevalent $\mathrm{CVD},{ }^{27,28}$ or mortality from CVD. ${ }^{19,29}$ Both structural support and functional support have been found to have associations with health outcomes. Functional social support, the type of support used in this research, is generally theorized to affect health by either promoting stress relief by buffering stressful life events ${ }^{20,30-32}$ (stress coping perspective) or by affecting self-esteem which can lead to better health behaviors (social constructionist or social cognitive model). ${ }^{33,34}$ According to the stress coping perspective, an individual's appraisal of a situation affects the relationship between stress and health. Some research has focused on perceived social support, ${ }^{11,35-38}$ whereas other studies have used measures of received functional support. ${ }^{39-41}$ Received support is confounded by need and used less frequently. Figure 1 presents a typical view in the social support literature of how social support has been conceptualized for research studies.

Individuals with higher levels of social support have a lower risk for developing $\mathrm{CVD}^{36-38,42-44}$ and those who have a history of CVD, who have higher levels of support, have better health outcomes. ${ }^{11,45-48}$

In addition, studies have suggested that social support is inversely associated with mortality ${ }^{23,28}$ and while the association is established, no large-scale studies have been done in postmenopausal women. Early studies investigating perceived social support's association with CVD were done only in men. ${ }^{36,38,42}$ More recently, both women and men were studied. However, in studies in both populations, the effect size was larger men, ${ }^{43,49}$ women, ${ }^{46}$ or different in men than in women, ${ }^{37}$ which indicates continued study of social support's effect on CVD and mortality in women is warranted. Notably, many of the former studies used significantly smaller cohorts. ${ }^{36-38,42}$ Despite there being a large body of research showcasing evidence of association of social ties to CVD, only a few studies used the social support measure of perceived support, instead using measures of structural support-social network scores or social ties. ${ }^{27,29}$ Therefore, further study of the relationship between perceived social support and CVD will help elucidate if the relatively strong association between functional social support and CVD in men is similar to that in women, and will help clarify whether lack of social support affects cardiovascular and overall mortality in postmenopausal women.

The purpose of this investigation was to assess perceived social support and its relationship to CVD or all-cause mortality in this large racially diverse cohort of postmenopausal women in the Women's Health Initiative Observational Study (WHI-OS). 


\section{METHODS}

\section{Study design and participants}

The WHI study design has been previously described in detail. ${ }^{50}$ Investigators planned for a prospective observational study of postmenopausal women to assess health risks and disease prevention longitudinally. WHI recruited more than 160,000 US women at 40 clinical centers between the ages of 50 and 79 years between 1993 and 1998 into 1 of 3 clinical trials or into the observational study. At the time of enrollment, baseline data were collected including physical measures such as blood pressure. Participants self-reported on their medical and psychological histories and answered multiple behavioral and psychological questions. Adjudicated health outcomes ${ }^{51}$ were available for women followed up to 10.8 years. Women who were not interested in or eligible to be in the clinical trial, in which dietary modification, hormone therapy, and/or calcium/vitamin D were being investigated, were asked to be a part of the observational study. General exclusions for participating in the WHI-OS were (1) not planning to live in the area for more than 3 years; (2) low likelihood of living for longer than 3 years; and (3) certain chronic diseases such as dementia or alcohol/drug dependency that would make participation difficult. ${ }^{52}$ The cohort of 93,676 women $^{52,53}$ was $83.3 \%$ White, $8.2 \%$ Black, 3.9\% Hispanic, and $2.9 \%$ Asian/Pacific and as such is considered ethnically and racially diverse. After excluding 961 women missing either the social support measure or adjudicated outcomes, the analysis sample for this study consisted of 92,715 women. An additional 1,943 women were excluded from analyses which stratified by prior CVD because of missing data on that variable.

\section{Demographic measures}

Demographic data including age, race, education, family income, and marital status were measured by self-report using questionnaires at baseline. ${ }^{50}$ Education was categorized from 0 to 8 years to college degree or higher. Participants indicated their family income as ranging from less than $\$ 10,000$ to more than $\$ 75,000$ per year. Marital status categories were (1) never married, (2) divorced/separated, (3) widowed, or (4) presently married/living as married.

\section{Psychosocial measures}

Nine support items that assessed perception of functional support were selected from the 19-item Medical Outcomes Study Social Support Survey (MOS-SSS). ${ }^{54}$ Sherbourne and Stewart ${ }^{54}$ devised the MOS-SSS, which was well-informed by theory and determined to be easy to administer, to assess social support in persons that are chronically ill. Investigators focused on the measurement of perceived availability of functional support. The MOS-SSS was developed to assess five dimensions of support: (1) emotional ("the expression of positive affect; empathetic understanding; and the encouragement of expressions and feelings"); (2) informational ("the offering of advice, information, guidance or feedback"); (3) tangible ("“،"،"'the provision of material aid or behavioral assistance"); (4) positive social interaction ("the availability of other persons to do fun things with you"); and (5) affectionate support ("involving love and affection"). Each of nine questions used in WHI asked participants to rank support from 1 to 5 on a Likert scale, where 1 was "none of the time" to 5 "all of the time". 55 A sample question reads: "How often is each of the following kinds of support available to you if you need it?: "Someone to have a good time with" (measuring positive social interaction) ${ }^{54}$ Because the WHI used only nine questions, there were too few items to produce highly reliable subscale scores for each dimension. Therefore, a total summary score of perceived social support was used instead. The summary score ranged from 9 to 45 , where the higher score corresponds to higher social support. Other WHI studies have used the social support measure. ${ }^{55,56}$ We found a coefficient alpha reliability of 0.93 in our sample, indicating high internal consistency. For testing statistical associations, we used social support as a continuous variable, but for descriptive purposes, to help convey magnitudes of association, we categorized social support in tertiles.

\section{Health behavior and dietary measures}

Health behaviors and dietary measures were assessed via self-report questionnaires. Smoking was categorized as never, past, or current. Alcohol intake was measured by asking participants how many servings they had per week. Participants were asked about the level of their walking and physical activity (quantified as mild, moderate, or strenuous). These responses were then translated into total Metabolic Equivalent of Task score for each week and were reported in quintiles. Dietary measures were the following: percentage saturated fat, percentage trans saturated fat, percentage sugar, and daily fruits and vegetables, all measured by a food frequency questionnaire. ${ }^{57}$

\section{Additional covariates}

Physical measurements included body mass index (BMI) and waist and hip circumferences for the waist to hip ratio and were assessed by clinical staff at baseline. Hormone therapy status was measured via questions asking about former and current hormone therapy use at baseline. Age at menopause which was estimated by age at last menstrual period or age at which last ovary was removed (indicating surgical menopause).

\section{Ascertainment of endpoints}

The primary outcomes for this study were (1) incident coronary heart disease (CHD), defined as myocardial infarction (MI) and/or death from CHD; (2) incident total CVD, defined as MI, stroke, or death caused by definite or possible CHD, cerebrovascular disease, or other CVD; and (3) allcause mortality which includes CVD mortality, cancer mortality, and non-CVD, noncancer mortality. Cardiovascular outcomes were adjudicated at local sites by investigators after review of semiannual self-administered medical history questionnaires and diagnoses were assigned at the local 
level. ${ }^{51}$ Myocardial infarction was established after physician review of questionnaires and subsequent medical record confirmation of electrocardiogram changes, elevated cardiac enzyme or troponin levels, or both. Stroke was documented in participants who suffered a neurological deficit appearing to have been related to a disruption of the brain arterial system, lasting more than 24 hours. Adjudicators assigned CHD as cause of death when CHD was specified on death certificate or autopsy report. Investigators used the National Death Index and follow-up was provided by family members regarding death status. ${ }^{51}$

\section{Statistical analysis}

Cox proportional hazards regression models were used to evaluate the relationships between social support and each of the three study outcomes. Social support was assessed as a continuous variable despite being categorized in tertiles for presentation. Time until each outcome was calculated by subtracting date of enrollment in the study from the event date or last day of follow-up. For models predicting the two cardiovascular outcomes, incident CHD, and incident total CVD, participants not experiencing either outcome were coded as censored when they were last observed to be alive. Martindale residual plots were used to assess the proportional hazard and linearity assumptions of social support for each outcome.

For each outcome, a Cox regression model was estimated first with social support as the sole predictor, followed by a model adding the following covariates to address potential confounding: age, education, race, income, and marital status, and select CVD risk factors (BMI, waist/hip ratio, smoking, physical activity, alcohol, hormone therapy, age at menopause, percentage saturated fat intake, percentage trans-saturated fat intake, percentage sugar intake, and fruit/vegetable intake). These well-established sociodemographic and behavioral factors are known to be determinants of CVD and allcause mortality. All covariates were selected a priori; no datadriven model selection approaches were employed. The sample was stratified by whether the participant reported a history of CVD before study enrollment.

To facilitate the interpretation of hazard ratios (HRs), social support was transformed to have a standard deviation of 1.0 in the full sample. One standard deviation corresponds to 7.92 points, and the scale ranges from 9 to 45 . Therefore, for example, a change from 9 points to 16.92 points for social support would correspond to a one standard deviation difference. All analyses were conducted using SAS statistical software, Version 9.3 (SAS Institute, Cary, NC).

\section{RESULTS}

The baseline characteristics of participants in the total sample $(n=92,715)$ and by perceived social support tertile categories are presented in Table 1 (see also Table, Supplemental Digital Content 1 [http://links.lww.com/MENO/ A373], which shows detailed descriptions of participant characteristics and outcome events by social support level). Unadjusted incident CHD was higher in those with lower support. A higher percentage of participants in the lower support group were diagnosed with CVD during the course of the study as compared to those reporting high support. Similarly, a higher percentage of those in the low support group died as compared to those in the high support group.

Of the potential confounding variables, income and marital status showed the strongest association with social support. For example, $27 \%$ of the women in the highest tertile of social support reported incomes greater than $\$ 75,000$ a year, as compared to $13 \%$ of women in the lowest tertile of support. Women in the highest tertile of social support were likely to be married (78\%). Of these women reporting higher support, $19 \%$ were divorced while out of the women in the lower support tertile, $50 \%$ were divorced. Of those in the lowest tertile of support, $10 \%$ were black and 5\% Hispanic/Latino as compared to $6 \%$ and $3 \%$, respectively of the women in the higher tertile of support. Participants in the lowest tertile of support were more likely to have high BMIs- $29 \%$ of women with BMI higher than 30 reported low support, whereas just $22 \%$ of those in the higher tertile of support had BMIs of more than 30. Participants in the higher tertile of support tended to have slightly higher educational attainment and were more likely to have indicated stronger emotional wellbeing. Those in the highest tertile of support scored on average 84 out of 100 points on the emotional wellbeing scale, whereas those in the lower tertile of support scored 72 points $(P<0.0001)$.

\section{Prediction of outcomes}

During the 10.8 years of follow-up in the 92,715 women, there were 2,060 cases of incident CHD (1,200 without prior CVD, and 850 with prior CVD). Total CVD cases were 4,440 (2,730 without prior CVD, and 1,710 with prior CVD). Cases of all-cause mortality were 6,029 (4,030 without prior CVD, and 1,999 with prior CVD).

Cox regression analyses were done separately for women with and without self-reported CVD at baseline (Table 2). Because 1,943 women had missing data on prior CVD, they were excluded from these analyses. Among women without prior CVD, who were the majority of the cohort $(n=73,421)$, the unadjusted HR for incident CHD per one standard deviation increase in social support was 0.92 (95\% confidence interval [CI], 0.87-0.97), indicating an estimated decrease in risk of incident heart disease of $8 \%$ for each standard deviation increase in social support. For total CVD, the HR was 0.86 (95\% CI, 0.83-0.89), whereas for overall mortality the risk was 0.83 (95\% CI, 0.81-0.86). When adjusted for potential confounders, the associations were substantially attenuated. For example, for incident CHD and total CVD, the HRs were 0.99 (95\% CI, 0.93-1.06) and 0.96 (95\% CI, 0.92-1.00), respectively. For all-cause mortality, the relationship attenuated to 0.95, but remained statistically significant (CI, 0.91-0.98).

Among women with prior CVD, the unadjusted HR for incident CHD per one standard deviation increase in social support was 0.93 (CI, 0.88-1.00), whereas for total CVD the unadjusted $\mathrm{HR}$ was 0.91 (95\% CI, 0.87, 0.95). The all-cause mortality unadjusted HR was 0.88 (95\% CI, $0.85,0.92)$. All of 
TABLE 1. Participant characteristics and outcome events by social support level

\begin{tabular}{|c|c|c|c|c|}
\hline & \multicolumn{3}{|c|}{ Perceived social support tertile } & \multirow[b]{2}{*}{ Total sample } \\
\hline & Low $(9-33)$ & Middle (34-40) & High (41-45) & \\
\hline Participants, $\mathrm{n}^{a}$ & 30,394 & 31,077 & 31,244 & 92,715 \\
\hline \multicolumn{5}{|c|}{ Self-reported cardiovascular disease prior to study entry, $\mathrm{n}(\%)$} \\
\hline Yes & $6,332(21)$ & $5,735(19)$ & $5,285(17)$ & $17,351(19)$ \\
\hline Unknown & 744 & 594 & 605 & \\
\hline \multicolumn{5}{|c|}{ Diagnosed with cardiovascular disease after study entry, $\mathrm{n}(\%)$} \\
\hline No & $29,439(97)$ & $30,306(98)$ & $30,512(98)$ & $90,257(97)$ \\
\hline Yes & $955(3)$ & $771(2)$ & $732(2)$ & $2,458(3)$ \\
\hline \multicolumn{5}{|l|}{ Died (all cause), n (\%) } \\
\hline No & $27,895(92)$ & $29,132(94)$ & $29,479(94)$ & $86,506(93)$ \\
\hline Yes & $2,499(8)$ & $1,945(6)$ & $1,765(6)$ & $6,209(7)$ \\
\hline \multicolumn{5}{|l|}{ Age at screening } \\
\hline Mean (SD) & $64.2(7.5)$ & $63.4(7.3)$ & $63.3(7.2)$ & $63.6(7.4)$ \\
\hline Some college or degree & $14,643(49)$ & $14,712(48)$ & $14,823(48)$ & $44,178(48)$ \\
\hline Some post-graduate or degree & $8,524(28)$ & $9,896(32)$ & $9,722(31)$ & $28,142(31)$ \\
\hline Unknown & 262 & 262 & 226 & \\
\hline \multicolumn{5}{|l|}{ Race, n (\%) } \\
\hline American Indian or Alaskan Native & $181(0.6)$ & $131(0.4)$ & $98(0.3)$ & $410(0.4)$ \\
\hline Asian or Pacific Islander & $899(3)$ & $950(3)$ & $787(3)$ & $2,636(3)$ \\
\hline Black or African-American & $2,960(10)$ & $2,502(8)$ & $2,004(6)$ & $7,466(8)$ \\
\hline Hispanic/Latino & $1,559(5)$ & $987(3)$ & $908(3)$ & $3,454(4)$ \\
\hline White (not of Hispanic origin) & $24,258(80)$ & $26,110(84)$ & $27,087(87)$ & $77,455(84)$ \\
\hline Other or unknown ${ }^{c}$ & 537 & 397 & 360 & \\
\hline \multicolumn{5}{|l|}{ Income, n (\%) } \\
\hline$<\$ 20,000$ & $6,943(25)$ & $3,957(14)$ & $2,846(10)$ & $13,746(16)$ \\
\hline$\$ 20,000-\$ 34,999$ & $7,710(27)$ & $6,645(22)$ & $5,682(19)$ & $20,037(23)$ \\
\hline$\$ 35,000-\$ 74,999$ & $9,990(35)$ & $12,154(42)$ & $12,538(43)$ & $34,682(40)$ \\
\hline 30 or more & 8,649 (29) & $7,455(24)$ & $6,896(22)$ & $23,000(25)$ \\
\hline Unknown & 382 & 345 & 356 & \\
\hline \multicolumn{5}{|l|}{ Waist/hip ratio } \\
\hline Mean (SD) & $0.815(0.08)$ & $0.804(0.08)$ & $0.801(0.08)$ & $0.81(0.08)$ \\
\hline Unknown & 170 & 176 & 156 & \\
\hline Smoking status, n (\%) & & & & \\
\hline Never smoked & $14,592(49)$ & $15,675(51)$ & $16,336(53)$ & $46,603(51)$ \\
\hline Past smoker & $12,957(43)$ & $13,219(43)$ & $13,041(42)$ & $39,217(43)$ \\
\hline Current smoker & $2,356(8)$ & $1,809(6)$ & $1,548(5)$ & $5,713(6)$ \\
\hline Unknown & 489 & 374 & 319 & \\
\hline METS $^{e}$ per week, n (\%) & & & & \\
\hline$<7$ & $13,471(45)$ & $11,932(39)$ & $11,116(36)$ & $36,519(40)$ \\
\hline 7 Or more & $16,606(55)$ & $18,863(61)$ & $19,831(64)$ & $55,300(60)$ \\
\hline Unknown & 317 & 282 & 297 & \\
\hline Alcohol servings per week & & & & \\
\hline Mean (SD) & $2.20(6.1)$ & $2.54(5.1)$ & $2.77(5.3)$ & $2.51(5.2)$ \\
\hline Unknown & 82 & 66 & 55 & \\
\hline Hormone therapy, $\mathrm{n}(\%)$ & & & & \\
\hline Never used hormones & $9,828(33)$ & $8,934(29)$ & $8,954(29)$ & $27,716(30)$ \\
\hline Past hormone user & $6,847(23)$ & $6,448(21)$ & $5,987(20)$ & $19,282(21)$ \\
\hline Current hormone user & $13,202(44)$ & $15,157(50)$ & $15,700(51)$ & $44,059(48)$ \\
\hline Unknown & 517 & 538 & 603 & \\
\hline
\end{tabular}


TABLE 1 (Continued)

\begin{tabular}{|c|c|c|c|c|}
\hline & \multicolumn{3}{|c|}{ Perceived social support tertile } & \multirow[b]{2}{*}{ Total sample } \\
\hline & Low $(9-33)$ & Middle (34-40) & High (41-45) & \\
\hline \multicolumn{5}{|l|}{ Early menopause, n (\%) } \\
\hline Unknown & 1,549 & 1,190 & 1,104 & \\
\hline \multicolumn{5}{|c|}{ Percent calories from saturated fatty acid } \\
\hline Mean (SD) & $10.2(3.4)$ & $10.0(3.3)$ & $9.8(3.3)$ & $10(3.3)$ \\
\hline Mean (SD) & $3.9(2.9)$ & $3.8(2.7)$ & $3.6(2.6)$ & $3.8(2.8)$ \\
\hline Unknown & 1,497 & 1,044 & 1,057 & \\
\hline \multicolumn{5}{|l|}{ Dietary total sugars, $g$} \\
\hline Mean (SD) & $100.5(47.8)$ & $99.9(44.3)$ & $99.4(44)$ & $99.9(45.4)$ \\
\hline Unknown & 1,497 & 1,044 & 1,057 & \\
\hline \multicolumn{5}{|c|}{ Fruits and vegetables, median servings/day } \\
\hline Mean (SD) & $4.2(2.2)$ & $4.4(2.2)$ & $4.5(2.2)$ & $4.4(2.2)$ \\
\hline \multicolumn{5}{|l|}{ Emotional well-being } \\
\hline Mean (SD) & $72(16.5)$ & $79.6(13)$ & $84(11.6)$ & $78.6(14.7)$ \\
\hline Unknown & 533 & 360 & 297 & \\
\hline \multicolumn{5}{|l|}{ Hypertension, n (\%) } \\
\hline Never hypertensive & $18,812(63)$ & $20,579(67)$ & $21,281(69)$ & $60,672(67)$ \\
\hline Untreated hypertensive & $2,762(9)$ & $2,263(7)$ & $2,208(7)$ & $7,233(8)$ \\
\hline Treated hypertensive & $8,223(28)$ & $7,722(25)$ & $7,265(24)$ & $23,210(26)$ \\
\hline Unknown & 597 & 513 & 490 & \\
\hline \multicolumn{5}{|l|}{ Systolic blood pressure } \\
\hline Mean (SD) & $127.5(18.1)$ & $126.5(17.9)$ & $126.8(17.9)$ & $126.9(18)$ \\
\hline Unknown & 597 & 513 & 490 & \\
\hline \multicolumn{5}{|c|}{ Treated for high cholesterol, n (\%) } \\
\hline No & $24,855(84)$ & $25,994(85)$ & $26,295(86)$ & $77,144(85)$ \\
\hline Yes & $4,840(16)$ & $4,442(15)$ & $4,322(14)$ & $13,604(15)$ \\
\hline \multicolumn{5}{|l|}{ Treated for diabetes, $\mathrm{n}(\%)$} \\
\hline
\end{tabular}

CHD, coronary heart disease; METS, metabolic equivalent of task; SD, standard deviation.

${ }^{a}$ Includes participants with prior cardiovascular disease (CVD) $(\mathrm{n}=17,351)$, without prior CVD $(\mathrm{n}=73,421)$, and unknown prior CVD status $(\mathrm{n}=1,943)$.

${ }^{b}$ Myocardial infarction.

"Combined with "other" in statistical analyses.

${ }^{d}$ Combined with $\$ 20,000$ to $\$ 34,999$ (most common group) in statistical analyses.

${ }^{e}$ Metabolic equivalents.

${ }^{f}$ This variable was included here for descriptive purposes but not included in analyses because of missing data.

these results became nonsignificant when adjusted for confounders- the HR for incident CHD was 1.04 (95\% CI, 0.97, $1.12)$; for total $\mathrm{CVD}=1.0(95 \% \mathrm{CI}, 0.95,1.06)$; and for allcause mortality $=1.02(95 \% \mathrm{CI}, 0.97,1.07)$.
In order to explore which of the potential confounders may have accounted for most of the attenuation in social support HRs predicting total CVD, a set of Cox regression analyses was repeated, including for each model a single covariate in

TABLE 2. Hazard ratios and 95\% confidence intervals for predicting cardiovascular disease and all-cause mortality from social support

\begin{tabular}{lllll}
\hline Prior CVD & \multicolumn{1}{c}{ Covariates } & Incident CHD $^{a}$ & Total CVD $^{b}$ & All-cause Mortality $^{c}$ \\
\hline No $(\mathrm{n}=73,421)$ & None & $0.92(0.87,0.97)$ & $0.86(0.83,0.90)$ & $0.83(0.81,0.86)$ \\
& Potential confounders & \\
& None & $0.99(0.93,1.06)$ & $0.96(0.92,1.00)$ & $0.95(0.91,0.98)$ \\
Yes $(\mathrm{n}=17,351)$ & Potential confounders $^{d}$ & $0.93(0.88,1.00)$ & $0.91(0.87,0.95)$ & $0.88(0.85,0.92)$ \\
& $1.04(0.97,1.12)$ & $1.00(0.95,1.06)$ & $1.02(0.97,1.07)$ \\
\hline
\end{tabular}

CHD, coronary heart disease; CVD, cardiovascular disease.

${ }^{a}$ Number of events: Incident CHD (myocardial infarction (MI) or death from CHD)-1,210 without prior CVD, 850 with prior CVD.

${ }^{b}$ Number of events: Total CVD (MI, stroke, and/or death from coronary heart disease, cerebrovascular disease, possible coronary heart disease, or other cardiovascular disease) - 2,730 without prior CVD, 1,710 with prior CVD.

${ }^{c}$ Number of events: All-cause mortality: 4,030 without prior CVD, 1,999 with prior CVD.

${ }^{d}$ Potential confounders: Accounted for sociodemographic characteristics (age, education, race, income, and marital status) and select traditional CVD risk factors (BMI [body mass index], waist/hip ratio, smoking, physical activity, alcohol, hormone therapy, age at menopause, percentage saturated fat intake, percentage trans-saturated fat intake, percentage sugar intake, and fruit/vegetable intake). 
TABLE 3. Cox regression hazard ratios for predicting total cardiovascular disease from social support among participants with no prior cardiovascular disease ${ }^{a}$ ( $n=73,421$; cardiovascular disease events $=2,730$ )

\begin{tabular}{lc}
\hline $\begin{array}{l}\text { Covariate in model } \\
\text { (in addition to social support) }\end{array}$ & $\begin{array}{c}\text { Hazard ratio for social } \\
\text { support }(95 \% \mathrm{CI})\end{array}$ \\
\hline No covariates & $0.87(0.83,0.89)$ \\
All primary covariates & $0.96(0.92,1.01)$ \\
Age & $0.91(0.88,0.95)$ \\
Education & $0.88(0.85,0.92)$ \\
Race & $0.87(0.83,0.90)$ \\
Income & $0.94(0.91,0.98)$ \\
Marital status & $0.92(0.88,0.96)$ \\
BMI category & $0.88(0.84,0.91)$ \\
Waist hip ratio & $0.88(0.84,0.90)$ \\
Smoking status & $0.87(0.84,0.91)$ \\
Exercise & $0.88(0.85,0.92)$ \\
Alcohol use & $0.87(0.83,0.90)$ \\
Hormone therapy & $0.88(0.85,0.92)$ \\
Early menopause & $0.87(0.84,0.91)$ \\
Percentage calories from saturated fatty acids & $0.86(0.83,0.89)$ \\
Dietary total trans fatty acid & $0.86(0.82,0.89)$ \\
Dietary total sugars & $0.85(0.82,0.89)$ \\
Fruits and vegetables & $0.86(0.83,0.89)$ \\
\hline
\end{tabular}

BMI, body mass index.

${ }^{a}$ Each row represents a separate model predicting total cardiovascular disease (CVD) from social support and the covariate listed in the first column.

the model in addition to social support. Analyses were conducted only in the group that did not report prior CVD at study entry. These data serve as an example of which covariates influenced the results. As shown in Table 3, the greatest attenuation produced by a single covariate occurred in the models with income, marital status, and age, in that order.

\section{DISCUSSION}

In this large diverse cohort of postmenopausal women from across the United States, we found that, in unadjusted analyses, higher levels of perceived social support were associated with a lower risk of incident CHD, total CVD, and all-cause mortality. This conclusion is consistent with other findings in the literature based on other populations. ${ }^{11,23,28,36-38}$ Furthermore, we found that higher levels of perceived support were related to lower CHD, CVD, and mortality both in women with and without CVD at baseline. However, the associations between perceived support and studied outcomes were not statistically significant after controlling for sociodemographic and lifestyle/behavioral factors, except for mortality in those without prior CVD.

We used a subset of questions from the MOS-SSS, which measures perceived functional support, and evaluated this subset's association with CVD and mortality. Other studies have used measures of functional support, ${ }^{36-38}$ but this very large cohort study is one of the largest to date to specifically study the relationship of perceived social support questions to CVD. Previous research has broadly showcased social support's association with CVD, but many studies have focused not on perceived social support but instead on structural measures of support, such as social network ties and integration. ${ }^{19,58}$ Perception of support is hypothesized to influence an individual's neuroendocrine system and buffer stress. ${ }^{49}$ Therefore, an individual who perceives she has support may be affected by physiological changes, which would protect her from CVD. However, in this longitudinal study of 10.8 years, we did not find an association between higher support and less CVD. A previous study in which adolescent Finns were surveyed on psychosocial factors and evaluated 27 years later demonstrated that a positive social environment as an adolescent contributes to better cardiovascular health. ${ }^{59} \mathrm{We}$ may have captured social support later in the lifetime of a woman than is relevant to development of CVD.

Some studies suggest that there may be a minimum threshold of social support, ${ }^{20,60}$ but our analyses showed a largely linear association of the scale we used with outcome HRs. This finding in this large cohort contributes to the theory regarding perceived support's influence on CVD and allcause mortality.

The potential mechanisms by which perceived social support influences CVD and other adverse outcomes is complex. There may be many influences on the outcomes that are difficult to extrapolate, so it is important to control for variables that may influence social support, CVD, or both. Social support can influence behaviors, ${ }^{26}$ and we controlled for the confounders of smoking, physical activity, alcohol use, and diet. However, the relationships on behaviors and support may be bidirectional, which makes the relationship complex. Although we found that social support was associated with a reduced risk of CVD outcomes and death, the findings were nearly completely eliminated when adding in confounding variables. In addition, while we controlled for a large number of cardiovascular risk factors, we acknowledge that not all potential confounders affecting CVD were controlled for. For example, since the study was designed and completed, inflammatory markers such as C-reactive protein and interleukin-6 are more commonly assessed in studies on CVD. We controlled for family income while other studies did not. ${ }^{36-38}$ The large number of covariates included in our analyses provided more rigorous control for confounding than in other studies. Other research has demonstrated social support's association with $\mathrm{MI},{ }^{37}$ coronary morbidity, ${ }^{36}$ and $\mathrm{CHD},{ }^{38}$ but none of these studies controlled for income, arguably our strongest confounding variable. Given our ability to control for a full range of confounders, our findings call into question prior results.

Women who had prior CVD were analyzed separately from those without prior CVD. Contrary to earlier reports, we did not find an association between social support and incident CHD or total CVD in women with or without prior CVD, but we did find a small inverse association between perceived support and all-cause mortality in women without prior CVD, who were in the majority. This may suggest that social support's influence on mortality differs between those with CVD versus those who are free of CVD; this issue needs further exploration. In addition, we acknowledge that social support could be confounded with self-care, in that women with higher social support have extra support to get to medical appointments and perform healthy behaviors such as exercise. Messina et $\mathrm{al}^{55}$ reported that women with higher support were more likely to access breast cancer screening. 
Our study contributes to the literature by showcasing use of functional support, using perception of support as the measure. Other studies assessed the relationship of social support (social ties) with mortality. ${ }^{23,28}$ Our findings suggest that the association between perceived social support and CVD in postmenopausal women is modest and that the confounding had a small effect on this association. One study evaluating the association of the psychosocial variables of anger, depression, and perceived social support found that persons with anger proneness and with depression had higher chances of developing peripheral artery disease while followed over 9.7 years. ${ }^{61}$ However, this same study did not show an association between low social support and peripheral artery disease. We found similar in that low social support did not appear to influence cardiovascular disease, like peripheral artery disease, another vascular disease. Many studies that show the association between low support and CVD indicate a trend for this relationship; however, continued exploration of this topic is of interest since study findings are conflicting.

Early studies on social support's effect on CVD were performed in cohorts that were solely male ${ }^{36,38}$ while others investigated both sexes but reported different responses to social support. In a large Japanese cohort study, ${ }^{43}$ $(\mathrm{n}=44,152)$ low perception of social support was strongly associated with stroke mortality in men $(\mathrm{HR}=1.59 ; 95 \% \mathrm{CI}$, 1.01-2.51), but the association was nonsignificant in women. Another study showed the opposite ${ }^{37}$-low social support was associated with increased risk of $\mathrm{MI}(\mathrm{HR}=2.72 ; 95 \% \mathrm{CI}$, 1.42-5.22) and stroke $(\mathrm{HR}=1.80,95 \% \mathrm{CI}, 1.05-3.10)$ in women but results were nonsignificant in men. In another large Japanese cohort study, ${ }^{62}$ researchers studied the psychosocial factor of living in multigenerational households as it relates to CHD and mortality. Although the Japanese study did not measure social support, but social interaction, it found that women living in multigenerational households had two to three times higher risk of CHD than did women who lived only with their spouses. Early on, researchers suspected that there may be sex differences related to the health effects of social support. ${ }^{21,63}$ However, many of these studies including ours were completed more than 10 years ago. We speculate that when early research was done, men and women had more traditional roles, and men might have had access to more support from women than women did from men. In recent years, the balance has been shifting and men may be giving support equal to that given by women. It is difficult to project whether the findings would be true today as traditional roles of men and women are everchanging. In addition, future studies should look at support levels in same-sex couples.

The WHI-OS cohort was interviewed at baseline 20 years ago, and the outcomes were assessed 10 years ago. Because CVD rates have increased in women in the last few decades, ${ }^{1}$ a similar study done today may have different results. In addition, since the initial assessment of social support in this cohort, online and social media support outlets such as Facebook have become commonplace, perhaps changing the way that support is gleaned and assessed by individuals. According to the Pew Research Center, 34\% of those older than 65 years use social media such as Facebook or Twitter. ${ }^{64}$ However, recent research shows that, converse to what might be expected, Facebook may decrease perceived support. ${ }^{65}$ And in a study on a Facebook measure of social support, researchers found that access to Facebook did not decrease depression and did not improve quality of life in study participants. ${ }^{66}$ Further studies my help determine whether online support is a valid type of social support.

Strengths of our study include the large sample size, the adjudicated outcomes, and the relative racial/ethnic diversity of the study sample. Follow-up was extensive, and attempts to capture missing data were prioritized. ${ }^{51}$ We controlled for a large number of confounders which can be seen as a strength of our study or a limitation as there could have been a flaw in our theory that the chosen covariates influenced the relationship.

All of these results, including ours, point to the need for further research on social support and its relation to CVD. Our large prospective cohort study in women contributes to the literature by showing that social support's relationship to CVD in women needs further clarification, and that there may be a small, but significant association between social support and mortality in women who do not have prior CVD.

\section{Study limitations}

Participation bias could have influenced the outcomes in that volunteers in the WHI could have been more healthy or different than the general population. The WHI researchers made a great effort to recruit a diverse cohort from across the United States, yet women who had the time and freedom to participate might have different characteristics than other women.

Although many potential confounding variables were controlled statistically, some residual confounding is likely. In particular, measures of inflammation, such as C-reactive protein and interleukin-6 were not yet seen as risk factors for CVD when the study was designed.

Social support was captured only at baseline of this study in which participants were followed for up to 10.8 years. We were thus unable to account for support at time of outcome or evaluate any relationship between change in social support, lifestyle or clinical variables, or outcomes.

In addition, there is no criterion standard for measuring social support in relation to health outcomes. This study used a common approach by measuring perceived social support but did not measure social ties, another common measure of support.

Most studies relating social support to CVD have assessed "emotional", or "attachment" support, ${ }^{38-40,45}$ a type of functional perceived support. We assessed emotional/informational support as well as tangible support, positive social interaction, and affection using a composite perceived support scale. We used nine questions from the MOS-SSS which was developed to assess perceived support in chronically ill persons, ${ }^{54}$ and as such might not have been the ideal measure for our healthy cohort. At least one study which demonstrated a relationship between social support and CVD assessed 
support in a work setting, ${ }^{37}$ which may indicate that support received at work is especially protective against CVD.

We acknowledge that aspects of social well-being such as having children or being religious could overlap with social support. We did not include assessment of those measures in our model.

\section{CONCLUSIONS}

This is the largest observational study to date in women to evaluate perceived social support's association with CVD and all-cause mortality. The major finding of our current study is that although perceived social support may be associated with fewer cardiovascular outcomes and decreased all-cause mortality, after controlling for potential confounders, these associations either become much smaller in magnitude or disappear.

Our conclusion that perceived social support, a type of functional support, may be mildly inversely associated with mortality is important in that it suggests that this is a trend that needs further clarification and investigation. Future research should focus on refining measurement of psychological risk factors such as social support, determining whether there are sex differences in psychological influences on health, particularly cardiovascular health, and assessing how psychological factors can be modified to positively affect health. In addition, research using, and clarifying between, multiple measures of social support with longitudinal assessment, are needed before drawing stronger conclusions about the causal role of social support in the development or progression of CVD.

\section{REFERENCES}

1. Benjamin EJ, Virvani SS, Callaway CW, et al. Heart disease and stroke statistics-2018-update: a report from the American Heart Association. Circulation 2018; 137:e67-e492.

2. Thompson DR, Ski CF. Psychosocial interventions in cardiovascular disease-what are they? Eur J Prev Cardiol 2013;20:916-917.

3. Linden W, Stossel C, Maurice J. Psychosocial interventions for patients with coronary artery disease: a meta-analysis. Arch Intern Med 1996;156:745-752.

4. Hegeman A, Schutter N, Comijs H, et al. Loneliness and cardiovascular disease and the role of late-life depression. Int $J$ Geriatr Psychiatry 2018;33:e65-e72.

5. Valtorta NK, Kanaan M, Gilbody S, Ronzi S, Hanratty B. Loneliness and social isolation as risk factors for coronary heart disease and stroke: systematic review and meta-analysis of longitudinal observational studies. Heart 2016;102:1009-1016.

6. Chen SJ, Chang CH, Chen KC, Liu CY. Association between depressive disorders and risk of breast cancer recurrence after curative surgery. Medicine (Baltimore) 2016;95:e4547.

7. Creed F, Morgan R, Fiddler M, Marshall S, Guthrie E, House A. Depression and anxiety impair health-related quality of life and are associated with increased costs in general medical inpatients. Psychosomatics 2002;43:302-309.

8. Jaremka LM, Andridge RR, Fagundes CP, et al. Pain, depression, and fatigue: loneliness as a longitudinal risk factor. Health Psychol 2014;33:948-957.

9. Cauley JA, Smagula SF, Hovey KM, et al. Optimism, cynical hostility, falls, and fractures: the Women's Health Initiative observational study (WHI-OS). J Bone Miner Res 2017;32:221-229.

10. Stickley A, Koyanagi A, Koposov R, Schwab-Stone M, Ruchkin V. Loneliness and health risk behaviours among Russian and U.S. adolescents: a cross-sectional study. BMC Public Health 2014;14:366.
11. Berkman LF, Leo-Summers L, Horowitz RI. Emotional support and survival after myocardial infarction: a prospective, population-based study of the elderly. Ann Intern Med 1992;117:1003-1009.

12. Kim ES, Hagan KA, Grodstein F, DeMeo DL, De Vivo I, Kubzansky LD. Optimism and cause-specific mortality: a prospective cohort study. Am J Epidemiol 2017;185:21-29.

13. Shipley BA, Weiss A, Der G, Taylor MD, Deary IJ. Neuroticism, extraversion, and mortality in the UK Health and Lifestyle Survey: a 21-year prospective cohort study. Psychosom Med 2007;69:923-931.

14. Giltay EJ, Geleijnse JM, Zitman FG, Hoekstra T, Schouten EG. Dispositional optimism and all-cause and cardiovascular mortality in a prospective cohort of elderly Dutch men and women. Arch Gen Psychiatry 2004;61:1126-1135.

15. Smoller JW, Pollack MH, Wassertheil-Smoller S, et al. Panic attacks and risk of incident cardiovascular events among postmenopausal women in the Women's Health Initiative Observational Study. Arch Gen Psychiatry 2007;64:153-160.

16. Arbelaez JJ, Ariyo AA, Crum RM, Fried LP, Ford DE. Depressive symptoms, inflammation, and ischemic stroke in older adults: a prospective analysis in the cardiovascular health study. $J$ Am Geriatr Soc 2007:55:1825-1830.

17. Shah BM, Shah S, Kandula NR, Gadgil MD, Kanaya AM. Psychosocial factors associated with subclinical atherosclerosis in South Asians: the MASALA study. J Immigr Minor Health 2016;18:1317-1327.

18. McCurley JL, Penedo F, Roesch SC, et al. Psychosocial factors in the relationship between socioeconomic status and cardiometabolic risk: the HCHS/SOL sociocultural ancillary study. Ann Behav Med 2017;51:477-488.

19. Ramsay S, Ebrahim S, Whincup P, et al. Social engagement and the risk of cardiovascular disease mortality: results of a prospective populationbased study of older men. Ann Epidemiol 2008;18:476-483.

20. Cohen S, Underwood LG, Gottlieb BH, editors. Social Support Measurement and Intervention: A Guide for Social Scientists. New York: Oxford University Press; 2000.

21. Shumaker SA, Hill DR. Gender differences in social support and physical health. Health Psychol 1991;10:102-111.

22. Perkins JM, Subramanian SV, Christakis NA. Social networks and health: a systematic review of sociocentric network studies in low- and middleincome countries. Soc Sci Med 2015;125:60-78.

23. Becofsky KM, Shook RP, Sui X, Wilcox S, Lavie CJ, Blair SN. Influence of the source of social support and size of social network on all-cause mortality. Mayo Clin Proc 2015;90:895-902.

24. Shaya FT, Chirikov VV, Mullins DC, et al. E. Social networks help control hypertension. J Clin Hypertens 2013;15:34-40.

25. Loucks EB, Berkman LF, Gruenewald TL, Seeman TE. Relation of social integration to inflammatory marker concentrations in men and women 70-79 years. Am J Cardiol 2006;97:1010-1016.

26. Ford EC, Ahluwalia IB, Galuska DA. Social relationships and cardiovascular disease risk factors: findings from the third national health and nutrition exam survey. Prev Med 2000;30:83-92.

27. Rutledge T, Linke SE, Olson MB, et al. Social networks and incident stroke among women with suspected myocardial ischemia. Psychosom Med 2008;70:282-287.

28. Kawachi I, Colditz GA, Ascherio A, et al. A prospective study of social networks in relation to total mortality and cardiovascular disease in men in the USA. J Epidemiol Community Health 1996;50:245-251.

29. Rutledge T, Kenkre TS, Thompson DV, et al. Psychosocial predictors of long-term mortality among women with suspected myocardial ischemia: the NHLBI-sponsored Women's Ischemia Syndrome Evaluation. J Behav Med 2016:39:687-693.

30. Cobb S. Presidential Address-1976. Social support as a moderator of life stress. Psychosom Med 1976;38:300-314.

31. Cohen S, Wills TA. Stress, social support, and the buffering hypothesis. Psychol Bull 1985;98:310-357.

32. Kaplan BH, Cassel JC, Gore S. Social support and health. Med Care SUPP 1977;15:47-58.

33. Lakey B, Cassady P. Cognitive processes in perceived social support. J Pers Soc Psychol 1990;59:337-343.

34. Lakey B, Drew J. A social-cognitive perspective on social support. In: Pierce GR, Lakey B, Sarason IB, Sarason BR, editors. Sourcebook of Social Support and Personality. New York: Springer Science and Business Media; 1991. pp. 107-140.

35. Rodriguez C, Burg M, Di Tullio M, et al. Effect of social support on nocturnal blood pressure dipping. Psychosom Med 2008;70:7-12. 
36. Rosengren A, Wilhelmsen L, Orth-Gomer K. Coronary disease in relation to social support and social class in Swedish men. A 15 year follow-up in the study of men born in. Eur Heart $J$ 2004;25:56-63.

37. Andre-Petersson L, Engstrom G, Hedblad B, Janzon L, Rosvall M. Social support at work and the risk of myocardial infarction and stroke in women and men. Soc Sci Med 2007;64:830-841.

38. Orth-Gomer K, Rosengren A, Wilhelmsen L. Lack of social support and incidence of coronary heart disease in middle-aged Swedish men. Psychosom Med 1993;55:37-43.

39. Rackow P, Scholz U, Hornung R. Received social support and exercising: an intervention study to test the enabling hypothesis. Br J Health Psychol 2015;20:763-776.

40. Maunder RG, Nolan RP, Park JS, James R, Newton G. Social support and the consequences of heart failure compared with other cardiac diseases: the contribution of support received within an attachment relationship. Arch Cardiovasc 2015;108:437-445.

41. Fontana A, Diegnan T, Villeneuve A, Lepore S. Nonevaluative social support reduces cardiovascular reactivity in young women during acutely stressful performance situations. $J$ Behav Med 1999;22:75-91

42. Rose G, Kumlin L, Dimberg L, Bengtsson C, Orth-Gomer K, Cai X. Work-related life events, psychological well-being and cardiovascular risk factors in male Swedish automotive workers. Occup Med (Lond) 2006;56:386-392.

43. Ikeda A, Iso H, Kawachi I, Yamagishi K, Inoue M, Tsugane S; for the JPHC Study Group. Social support and stroke and coronary disease: the JPHC study cohorts II. Stroke 2008;39:768-775.

44. Kopp M, Skrabski A, Szanto Z, Siegrist J. Psychosocial determinants of premature cardiovascular mortality differences within Hungary. J Epidemiol Community Health 2006;60:782-788.

45. Wang HX, Mittleman MM, Orth-Gomer K. Influence of social support on progression of coronary disease in women. Soc Sci Med 2005;60:599607.

46. Krumholz HM, Butler J, Miller M, et al. Prognostic importance of emotional support for elderly patients hospitalized with heart failure. Circulation 1998;97:958-964.

47. Lurie I, Myers V, Goldbourt U, Gerber Y. Perceived social support following myocardial infarction and long-term development of frailty. Eur J Prev Cardiol 2015;22:1346-1353.

48. Weiss-Faratci N, Lurie I, Neumark Y, et al. Perceived social support at different times after myocardial infarction and long-term mortality risk: a prospective cohort study. Ann Epidemiol 2016;26:424-428.

49. Mezuk B, Diez Roux AV, Seeman T. Evaluating the buffering vs. direct effects hypotheses of emotional social support on inflammatory markers: the multi-ethnic study of atherosclerosis. Brain Behav Immun 2010;24:1294-1300.

50. The Women's Health Initiative Study Group. Design of the Women's Health Initiative Clinical Trial and Observational Study. Control Clin Trials 1998;19:61-109.
51. Curb JD, McTiernan A, Heckbert SR, Kooperberg C, Stanford J, Nevitt $\mathrm{M}$, et al. Outcomes ascertainment and adjudication methods in the women's health initiative. Ann of Epidemiol 2003;13:S122-S128.

52. Langer RD, White E, Lewis CE, Kotchen JM, Henrdrix SL, Trevisan M. The Women's Health Initiative Observational Study: baseline characteristics of participants and reliability of baseline measures. Ann Epidemiol 2003;13:S107-S121.

53. Hay J, Hunt JR, Hubbell FA, et al. The Women's Health Initiative recruitment methods and results. Ann of Epidemiol 2003;13:S18-S77.

54. Sherbourne CD, Stewart AL. The MOS social support survey. Soc Med 1991;32:705-714.

55. Messina CR, Lane DS, Glanz K, et al. Relationship of social support and social burden to repeated breast cancer screening in the Women's Health Initiative. Health Psychol 2004;23:582-594.

56. Kroenke $\mathrm{CH}$, Michael Y, Tindle H, et al. Social networks, social support and burden in relationships, mortality after breast cancer diagnosis. Breast Cancer Res Treat 2012;133:375-385.

57. Patterson R, Kristal A, Tinker L, Carter R, Bolton M, Agurs-Collins T. Measurement characteristics of the Women's Health Initiative food frequency questionnaire. Ann Epidemiol 1999;9:178-187.

58. Shumaker S, Czajkowski SM, editors. Social Support and Cardiovascular Disease. New York: Springer Science and Social Media; 2013.

59. Pulkki-Raback L, Elovainio M, Hakulinen C, et al. Cumulative effect of psychosocial factors in youth on ideal cardiovascular health in adulthood: the Cardiovascular Risk in Young Finns Study. Circulation 2015;131:245-253.

60. Mookadam F, Arthur H. Social support and its relationship to morbidity and mortality after acute myocardial infarction: systematic overview. Arch Intern Med 2004;164:1514-1518.

61. Wattanakit K, Williams JE, Schreiner PJ, Hirsch AT, Folsom AR. Association of anger proneness, depression and low social support with peripheral arterial disease: the Atherosclerosis Risk in Communities Study. Vasc Med 2005;10:199-206.

62. Ikeda A, Iso H, Kawachi I, et al. Living arrangement and coronary heart disease: the JPHC study. Heart 2009;95:577-583.

63. Bartley M, Martikainen P, Shipley M, Marmot M. Gender differences in the relationship of 6 partner's social class to behavioural risk factors and social support in the Whitehall II study. Soc Sci Med 2004;59:1925-1936.

64. Pew Research Center. Tech Adoption Climbs Among Older Adults. 2017. Available at: http://www.pewinternet.org/2017/05/17/tech-adoptionclimbs-among-older-adults/PI_2017.05.17_Older-Americans-Tech_FINAL\%20(1).pdf. Accessed December 17, 2018.

65. Lee E-J, Cho E. When using Facebook to avoid isolation reduces perceived social support. Cyberpsychol Behav Soc Netw 2018;21:32-39.

66. McCloskey W, Iwanicki S, Lauterbach D, Giammittorio DM, Maxwell K Are Facebook "friends" helpful? Development of a Facebook-based measure of social support and examination of relationships among depression, quality of life, and social support. Cyberpsychol Behav Soc Netw 2015;18:499-505. 